\title{
LA RELACIÓN DERIVATIVA
}

\author{
Jesús Pena \\ Universidad de Santiago de Compostela \\ Jepena@usc.es
}

\section{Resumen}

One of the main objectives of derivational morphology is the description of the relationship between form and meaning of words, what is called 'derivational relationship'. There are currently rather different opinions about the nature of this relationship. The aim of this article is to show the different levels in which derivational relationship can be described. The main problems that it causes, as well as some of the current solutions, will be reviewed.

La razón de ser de la morfología derivativa resulta de un hecho evidente: en el léxico de una lengua hay palabras que se relacionan formal y semánticamente. Un ejemplo claro de palabras así relacionadas es la denominada 'familia léxica', comp. blanco, blancuzco, blanquinegro, blancura, blanquear, blanqueo, etc. Lo que hace que tales palabras se integren en un mismo grupo es lo que tienen en común, la base de comparación, en este caso lo que denominamos la 'raíz'. El significante común, blanc-, presente en blanco, reaparece en las demás palabras del grupo con el mismo significado "blanco".

Lo compartido por dos o más palabras no tiene por qué ser necesariamente la raíz; puede ser otro segmento más amplio o un afijo. Así desestabilizar, desestabilizador y desestabilización comparten el tema complejo desestabiliza-; por otro lado, el significante -uzc-, presente en blancuzco con el significado aspectual "casi", reaparece con ese mismo significado en negruzo o parduzco; el significatnte -er-, presente en camionero con el significado "persona que realiza una actividad relacionada con lo designado por la palabra base", reaparece en relojero, zapatero, etc.

Precisamente, cuando analizamos morfológicamente una palabra partimos del postulado siguiente: la parte común a dos o más palabras consta de un determinado significante asociado a un determinado significado. Pues bien, una de las tareas centrales de la morfología derivativa es describir la relación entre las formas y los significados de las palabras, esto es, la denominada 'relación derivativa'. Es esta quizá la cuestión más importante teóricamente porque la elección del modo de concebir dicha relación determinará la forma de entender y formular las reglas de derivación y, en líneas más generales, el modelo de morfología que se adopte. El propósito de este artículo será mostrar los distintos niveles en que aparece la relación derivativa y ver los problemas que se plantean, así como algunas de las soluciones adoptadas.

En la actualidad existen interpretaciones bastante divergentes respecto de la naturaleza de la relación derivativa. La cuestión se centra en torno a las posibles discordancias entre la estructura morfológica de una palabra y su interpretación semántica. Y el problema que 
se plantea es saber si tales discordancias permiten o no estudiar conjuntamente las relaciones morfológicas y las relaciones semánticas. A este respecto se mantienen dos posturas antagónicas: la de quienes defienden la asociación entre la estructura formal y la estructura semántica, y la de quienes sostienen la disociación entre ambas estructuras. Las dos posturas son conocidas como la tesis asociativa y disociativa, respectivamente (vid. Corbin, 1987: 208-260 y Rainer 1989: 330-400).

$\mathrm{El}$ ámbito de esta relación entre la forma y el significado puede y debe ser estudiado en tres niveles o estratos: 1) entre dos o más palabras, 2) dentro de una misma palabra y 3) en el ámbito de un determinado afijo o, en general, de un determinado procedimiento de formación de palabras.

\section{Relación derivativa entre dos o más palabras}

En líneas generales, podemos encontrarnos con las cuatro situaciones siguientes:

(a) Palabras relacionadas formal y semánticamente: animar-animación; funcionar-funcionamiento.

(b) Palabras relacionadas formalmente pero no semánticamente: estar-estación, instruir-instrumento.

(c) Palabras relacionadas semánticamente pero no formalmente: juego-lúdico; grasa-adiposo.

(d) Palabras sin relación formal ni semántica: meter-misión, querer-cuestión.

La pregunta que se plantea ante estas cuatro situaciones es si, para poder hablar de relación derivativa entre la palabra base de derivación y la palabra derivada, debe haber una relación formal y semántica. Si la respuesta es afirmativa, solo hay relación derivativa en (a): entre las parejas animar-animación y funcionar-funcionamiento, porque hay relación formal y semántica; pero no hay relación derivativa en las tres situaciones restantes: en (b), entre estar-estación e instruir-instrumento, porque solo hay relación formal; en (c) entre, juego-lúdico, grasa-adiposo, porque solo hay relación semántica, y en (d), entre meter-misión, querer-cuestión, porque no hay relación formal ni semántica. En este supuesto, las relaciones derivativas serían siempre y a la vez formales y semánticas.

Estas discordancias entre el nivel morfológico y el nivel semántico se interpretan de diferentes maneras, fundamentalmente dos: 1) la relación derivativa es formal y semántica, en cuyo caso solo entra la situación (a);2) la relación derivativa es una relación formal, que puede ir acompañada de una relación semántica; en este caso entran las situaciones (a) y (b). Donde sí hay unanimidad es en eliminar del ámbito de la morfología derivativa no solo el caso (d), sino también el (c): las relaciones semánticas no asociadas a reglas formales (morfológicas) no son relaciones derivativas.

\section{Relación derivativa dentro de una misma palabra: la relación entre su estructura formal y semántica. Las paradojas del encorchetamiento}

El segundo ámbito donde se plantea el problema de la relación formal y semántica es en el interior de la palabra derivada o compleja. En general, el significado de una palabra 
derivada viene a ser el resultado de sumar al significado de la base (o de las bases si es una palabra compuesta) el significado que aporta una determinada regla de formación de palabras (RFP) (Rainer, 1993: 101). La suma de ambos significados suele expresarse en forma de una paráfrasis composicional. Por ejemplo, en la paráfrasis "acción de V" formulada para nombramiento $V$ representa una variable, el significado del verbo base, y "acción de" el significado que aporta la regla de sufijación con - miento. En la paráfrasis "acto propio de nombre de persona" aplicada a niñeria, "nombre de persona" representa el significado genérico de la base (una subclase de nombres) y "acto propio de" el significado que aporta la regla de sufijación con-ería.

Esta manera de interpretar el significado de la palabra derivada se conoce como el 'principio de la composicionalidad'. Se entiende por composicionalidad el hecho de que a la palabra derivada se le pueda asociar una paráfrasis regular donde figura la base de esa palabra. En la paráfrasis de la palabra nombramiento, "acción de nombrar", está nombrar; en la paráfrasis de niñería, "acto propio de un niño", está niño.

Del principio de la composicionalidad deriva el hecho de que la estructura semántica de una palabra compleja tiene que ser paralela a su construcción formal. Pero hay aquí otro tipo de irregularidad, que cuestiona el principio de la composicionalidad que acabamos de ver. Esta irregularidad, denominada 'paradoja del encorchetamiento' se refiere a la falta de correlación o correspondencia entre la estructura formal y la estructura semántica de la palabra compleja o derivada. En efecto, son frecuentes los casos donde la construcción formal o estructura jerárquica de la palabra derivada no se corresponde con su estructura semántica. La construcción formal y la construcción semántica de la palabra derivada no siempre concuerdan, no siempre son isomorfas o simétricas.

En la morfología derivativa del español hay, por ejemplo, un tipo de construcción donde ocurre manera sistemática este tipo de discordancia. Se trata de adjetivos que presentan la secuencia mórfica prefijo-sustantivo-sufijo. Los prefijos que figuran en tales adjetivos indican "adhesión u oposición", "relación locativa", "relación temporal" o "cuantificación". Veamos, a modo de ejemplo, los adjetivos antigripal, posconciliar y unifamiliar en los sintagmas vacuna antigripal ("contra la gripe"), iglesia posconciliar ("posterior al concilio"), vivienda unifamiliar ("de una familia"). La estructura formal o jerárquica de tales adjetivos es como sigue:

$\begin{array}{ll}\text { [anti [[grip] al] }] & \text { gripe } \rightarrow \text { gripal } \rightarrow \text { antigripal } \\ {[\text { pos }[[\text { concili] ar }]]} & \text { concilio } \rightarrow \text { conciliar } \rightarrow \text { posconciliar } \\ {[\text { uni }[[\text { famili }] \text { ar }]]} & \text { familia } \rightarrow \text { familiar } \rightarrow \text { unifamiliar }\end{array}$

Formalmente, pues, las bases a las que se adjuntan los prefijos son los adjetivos gripal, conciliar y familiar. Sin embargo, la relación semántica expresada por el prefijo no se refiere a todo el adjetivo, sino solo a su base nominal, como claramente se refleja en las paráfrasis indicadas. Así en vacuna antigripal ("contra la gripe"), la relación "contra" expresada por anti- se establece entre el sustantivo gripe, que es la base nominal del adjetivo gripal, y el sustantivo modificado vacuna; en iglesia posconciliar ("posterior al concilio"), el prefijo pos- expresa la relación temporal "posterior a" entre concilio, sustantivo base del adjetivo conciliar, y el sustantivo modificado iglesia, y en vivienda unifamiliar ("de una familia") es la base nominal de familiar -el sustantivo familia - la que resulta cuantificada por el prefijo $u n i^{-}$. 
Son varios las soluciones adoptadas respecto del tipo de formaciones aquí ejemplificadas y ninguna exenta de objeciones. Véanse, por ejemplo, Corbin (1980 y 1987: 121-139) o Rainer (1993: 101-104). Para una visión general de los problemas que plantea este tipo de discordancias denominadas paradojas de encorchetamiento, pueden consultarse Spencer (1991: 397-422) o Becker (1993).

\section{Relación derivativa en el ámbito de un determinado afijo o, en general, en el ámbito de un determinado procedimiento de formación de palabras}

Los ejemplos de la serie (1) nos muestran un fenómeno muy frecuente en la formación de palabras, conocido generalmente como 'asimetría morfológica' (Jackendoff, 1975: 650-652). Esto es, de un lado, un mismo afijo, por ejemplo, expresa distintos significados; de otro, a cada uno de dichos significados pueden corresponder varios afijos:

(1)

"gradación o intensificación máxima"

$\begin{array}{llll}\text {-ísimo } & \text { grandísimo } & \text { archi-: archisutil } & \text { larchi-: archiduque } \\ \text {-ón } & \text { grandón } & \text { extra-: extrafino } & \text { lextra-: extrarradio } \\ \text {-azo } & \text { grandazo } & \text { ultra-: ultramoderno } & \text { /ultra-: ultramar }\end{array}$

Para expresar la propiedad de un adjetivo en grado sumo, el español dispone de varios prefijos y sufijos; a su vez, los prefijos ejemplificados pueden expresar más de un significado: archi- puede significar también "jerarquía" (duque $\rightarrow$ archiduque), extra- la relación locativa "fuera de" (radio $\rightarrow$ extrarradio) y ultra- la relación locativa "más allá de" (mar $\rightarrow$ ultramar).

Es más, en esta misma línea, tal como se puede ver en (2), a una misma forma afijal corresponden distintos significados y distintas relaciones categoriales (RC) entre la palabra base y la palabra derivada:

$\begin{array}{lllll} & \mathrm{RC} & \text { significado } & -i a & \text { otros sufijos } \\ \text { a. } & \mathrm{A} \rightarrow \mathrm{S} & \text { cualidad } & \text { alegria } & \text { mediocridad, belleza, finura } \\ \text { b. } & \mathrm{S} \rightarrow \mathrm{S} & \text { colectivo } & \text { cofradia } & \text { electorado, ejemplario } \\ \text { c. } & \mathrm{S} \rightarrow \mathrm{S} & \text { locativo } & \text { abadia } & \text { confesionario, conejera } \\ \text { d. } & \mathrm{S} \rightarrow \mathrm{S} & \text { acto propio de } & \text { rateria } & \text { bribonada }\end{array}$

Merece la pena detenerse en estos hechos de falta de correspondencia entre forma y significado tan frecuentes en la morfología derivativa de nuestras lenguas. Los ejemplos están tomados del español, pero ocurren igualmente en cualquier lengua del mismo tipo morfológico.

Efectivamente, desde un punto de vista semasiológico a un mismo afijo pueden corresponder varios significados, comp. el sufijo - ería en 
(3)

$\begin{array}{llll} & \mathrm{RC} & \text { significado } & \\ \text {-ería } & \mathrm{A} \rightarrow \mathrm{S} & \text { cualidad } & \text { galanteria, tacañeria } \\ \text {-ería } & \mathrm{S} \rightarrow \mathrm{S} & \text { actividad } & \text { albañileria, ebanisteria } \\ \text {-eria } & \mathrm{S} \rightarrow \mathrm{S} & \text { lugar de actividad } & \text { marmoleria, cerveceria } \\ \text {-eria } & \mathrm{S} \rightarrow \mathrm{S} & \text { locativo/ubi } & \text { enfermeria, hospederia } \\ \text {-ería } & \mathrm{S} \rightarrow \mathrm{S} & \text { colectivo } & \text { chiquilleria, cuberteria } \\ \text {-eria } & \mathrm{S} \rightarrow \mathrm{S} & \text { acto propio de } & \text { niñeria, bellaquería }\end{array}$

A la inversa, desde un punto de vista onomasiológico, un mismo significado puede ser codificado por afijos distintos, comp. el significado "colectivo" en los siguientes sustantivos denominales $(\mathrm{S} \rightarrow \mathrm{S})$ :

$\begin{array}{ll}\text { (4) } & \\ \text {-ada } & \text { boyada, arcada } \\ \text {-ado } & \text { alumnado, teclado } \\ \text {-adura } & \text { botonadura, dentadura } \\ \text {-aje } & \text { andamiaje, cortinaje } \\ \text {-al } & \text { instrumental, personal } \\ \text {-amen } & \text { barrilamen, pelamen } \\ \text {-ario } & \text { epistolario, ejemplario } \\ \text {-era } & \text { cabellera, cajonera } \\ \text {-ería } & \text { chiquillería, cubertería } \\ \text {-erío } & \text { griterío, puterío } \\ \text {-ero } & \text { cancionero, refranero } \\ \text {-ía } & \text { cofradía, marinería } \\ \text {-ío } & \text { gentío, monjío }\end{array}$

Todos estos casos parecen subrayar la tesis de quienes defienden que en la representación de la relación derivativa hay que disociar la relación formal o morfológica y la relación semántica, que en las reglas de derivación hay que separar las reglas morfológicas y las reglas semánticas. Vid. Jackendoff (1975) o Lieber (1980).

\subsection{La noción de paradigma derivativo o tipo de derivación}

Hay, sin embargo, una manera de resolver en parte esta asimetría morfológica, este entrecruzamiento o superposición entre procedimientos de formación y significados. Y de esta manera trabajamos, de hecho, los que nos dedicamos al estudio de la morfología cuando queremos describir la morfología derivativa de una lengua particular. Es la siguiente: en aquellos casos en que a un mismo significado corresponden varios procesos o procedimientos morfológicos (concurrencia de afijos, afijación/composición, sufijación/prefijación, afijación/conversión, etc.), asociar a una sola regla de formación de palabras (RFP) aquellos procedimientos de formación que reúnan los dos requisitos siguientes: (a) que expresen un mismo significado, (b) que plasmen una relación categorial única entre la clase de la palabra base de derivación y la clase de la palabra derivada, comp. sustantivos deadjetivales, adjetivos denominales, etc.

Si releemos ahora los ejemplos de (2) bajo la nueva interpretación que exigen los dos requisitos mencionados, tendremos cuatro RFP: (a) la que crea sustantivos deadjetivales con 
el significado "cualidad de" mediante los sufijos -ia, -idad, -eza y -ura, (b) la que forma sustantivos denominales con significado "colectivo" por medio de la sufijación con - $i a$, -ado y -ario, (c) la que permite crear sustantivos denominales con el significado "locativo" mediante sufijación de -ia, -ario y -era, y (d) la que forma también sustantivos denominales con el significado "acto propio de" a través de la adjunción sufijal de -ia y -ada. La misma relectura se puede hacer con los casos ejemplificados en (3) y (4).

Esta manera de proceder responde a lo que se conoce como "modelo asociativo débil", tal como lo propone Corbin (1987: 233):

Dans les cas où à un même sens $\mathrm{S}$ correspondent plusieurs processus morphologiques $\mathrm{M}_{1}$, $M_{2}, M_{3} \ldots M_{n}$ (concurrence d'affixes, affixation et conversion, etc.), je propose d'associer à la $\mathrm{RCM}$ (= règle de construction de mots), caractérisée par $\mathrm{S}$, un paradigme d'opérations morphologiques comportant $M_{1}, M_{2}, M_{3} \ldots M_{n}$.

Aunque en la propuesta así formulada no aparece de manera explícita el requisito de la relación categorial única, Corbin lo tiene siempre en cuenta y aparece enunciado de forma explícita en el apartado de las conclusiones cuando define en términos ecuacionales la RCM, ahora denominada 'opération dérivationnelle':

Une règle de construction de mots (RCM) associe une structure morphologique mettant en jeu un rapport catégoriel (orienté) entre deux et seulement deux catégories lexicales semblables ou différentes, une opération sémantique et un ensemble d'opérations morphologiques. (Corbin, 1987: 257)

Una propuesta similar a la de Corbin ${ }^{1}$ es la que hace Zwanenburg (1984a: 138) bajo el término tipo de derivación, que define así:

By a derivation type I mean a set of derivation processes which are characterized by the use of bases of a given lexical category and of a set of suffixes of a given lexical category and which have the same global meaning.

La RFP (= RCM en Corbin) aparece así caracterizada por la asociación entre un significado + una relación categorial + uno o más afijos o procedimientos de formación.

Este conjunto de diversos procedimientos morfológicos asociados a una misma RFP lo denomina Corbin 'paradigma de operaciones morfológicas' o 'paradigma morfológico'. La RFP así caracterizada reçibe también otras denominaciones por parte de otros autores: 'tipo de derivación' (Zwanenburg, 1984a), 'categoría de formación de palabras' (Wortbildungskategorie) (Rainer, 1993), 'categoría derivacional' (Rainer, 1999), etc. En (5) tenemos un ejemplo de paradigma derivativo, el de los sustantivos deadjetivales que expresan "cualidad de":
$\mathrm{A} \rightarrow \mathrm{S}$
$-e r a$
$\mathrm{A} \rightarrow \mathrm{S}$
$-e z$
cojera, ceguera, ronquera
altivez, algidez, honradez

\footnotetext{
1 Corbin (1987: 233, nota 53) señala, sin embargo, varias diferencias entre su propuesta y la de Zwanenburg.
} 


$\begin{array}{lll}\mathrm{A} \rightarrow \mathrm{S} & -e z a & \text { belleza, grandeza } \\ \mathrm{A} \rightarrow \mathrm{S} & -i a & \text { audacia, demencia } \\ \mathrm{A} \rightarrow \mathrm{S} & -i a & \text { cobardia, osadia } \\ \mathrm{A} \rightarrow \mathrm{S} & -(\mathrm{V}) \text { dad } & \text { debilidad, mediocridad } \\ \mathrm{A} \rightarrow \mathrm{S} & - \text { itud } & \text { aptitud, exactitud } \\ \mathrm{A} \rightarrow \mathrm{S} & - \text { or } & \text { amargor, espesor } \\ \mathrm{A} \rightarrow \mathrm{S} & - \text { ura } & \text { anchura, locura } \\ \mathrm{A} \rightarrow \mathrm{S} & - \text {-ería } & \text { galanteria, tacañeria } \\ \mathrm{A} \rightarrow \mathrm{S} & - \text { ismo } & \text { paralelismo, salvajismo }\end{array}$

No todos los procesos morfológicos adscritos al mismo paradigma están igualmente disponibles (Corbin, 1987: 234). En primer lugar, hay diferencias en cuanto al grado de productividad; por ejemplo, en español hay una veintena de sufijos en el paradigma de los sustantivos deadjetivales, pero los que hoy están disponibles son: $-(V) d a d,-e z,-i a,-i a$, -ería e-ismo y, en mucho menor grado, -era, -eza, -ura-or e -itud. No están disponibles: -icie (canicie), -ie (barbarie) o-umbre (pesadumbre). En segundo lugar, factores de diversa naturaleza hacen que los afijos disponibles puedan actuar libremente sobre las bases en que operan (concurrencia afijal) o impongan algún tipo de restricción seleccionando bases con determinadas propiedades. En el paradigma de los sustantivos deadjetivales, no todos los sufijos actúan sobre adjetivos sufijados y el más expandido es $-(V) d a d$, que selecciona la mayoría de los adjetivos sufijados: casualidad, irritabilidad, europeidad, corporeidad, fangosidad, etc. En cambio, sobre adjetivos no sufijados la concurrencia afijal es mayor; de ahí la existencia de sustantivos deadjetivales derivados sobre la misma base y con el mismo significado, sancionados en el uso como comunes o no restringidos:

(6)

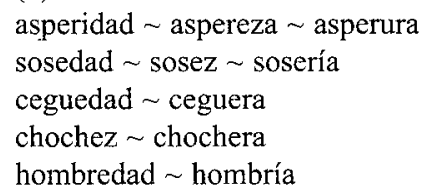

De la caracterización de la RFP que hace Corbin se deducen varias consecuencias. De entre ellas, destacaré dos que considero fundamentales:

(1) Hay tantas RFP diferentes cuantas asociaciones diferentes existen entre una relación categorial y un significado (Corbin, 1983: 257).

(2) De un lado, a una RFP pueden estar asociados varios afijos derivativos pero, de otro, un afijo derivativo dado no puede estar asociado más que a una sola RFP (ibid.). Lo que quiere decir que habrá tantos afijos homónimos cuantos significados distintos o relaciones categoriales diferentes correspondan a una misma forma afijal.

La noción de paradigma derivativo, tipo de derivación, etc. no resuelve, ni mucho menos, todos los casos de entrecruzamiento o de asimetría morfológica. Además, la solución homonímica, tal como queda planteada, resulta problemática.

En efecto, sostener que hay un nuevo afijo homónimo, siempre que aparezca un significado distinto o una relación categorial distinta entre la palabra base y la derivada, puede resultar antieconómico o simplemente inadecuado. Basta pensar en aquellas situaciones donde a un afijo no se asocian simultáneamente más de una $\mathrm{RC}$ y más de un significado 
(vid. Corbin, 1987: 242-254). En esquema, pueden aparecer las dos situaciones ejemplificadas en (7) y (8):

(7)

$\begin{array}{ll}\text { un afijo } & \text { una } \mathrm{RC} \\ - \text { dor } & \mathrm{V} \rightarrow \mathrm{S} \\ - \text { dor } & \mathrm{V} \rightarrow \mathrm{S}\end{array}$

(8)

$\begin{array}{ll}\text { un afijo } & \text { varias RC } \\ -i z-a-r & \mathrm{~A} \rightarrow \mathrm{V} \\ -i z-a-r & \mathrm{~S} \rightarrow \mathrm{V}\end{array}$

varios significados

agente: decorador, programador

instrumento: congelador, programador

En (7) tenemos el caso en que a una misma forma afijal corresponden una misma relación categorial y varios significados. Si las palabras derivąas con una misma forma afijal pueden tener dos o más significados asociados a una misma relación categorial, cabe pensar en la posibilidad de que estas palabras se construyan mediante la misma RFP con la ayuda de un mismo afijo, y no deducir de manera casi automática que estamos ante afijos homónimos. La cuestión previa que hay que resolver -más propia de la semántica que de la morfología- es si las diferencias de significado encontradas son invariantes o pueden ser interpretadas como variantes, bien en términos de una invariante más abstracta, bien en términos de significados relacionados, de los cuales uno es el básico o nuclear y los demás secundarios o derivados del primario.

En (7) vemos que el sufijo - dor puede expresar los significados "agentivo" e "instrumental". En principio, estos significados parecen ser diferentes; pero resulta que hay nombres en -dor con significado agentivo (decorador, diseñador, constructor) con significado instrumental (congelador, grapadora, secador secadora) y con ambos significados -agentivo e instrumental- (agitador, programador, soldador).

Según Rio-Torto (1998), el hecho de que nombres como agitador, distribuidor, programador o soldador admitan una interpretación como agentivos humanos y como instrumentos habla a favor de unificar en una sola RFP la construcción de agentivos humanos ("el que V") y de nombres de instrumento ("aquello con que se V"). Añade la autora:

Nesta linha de pensamento, nada obsta a que, virtualmente, guiador ou regador possam designar um ser humano que tem por função $\mathrm{V}$, ou um objecto que desempenha o mesmo papel. Assim, o carácter [ \pm humano] ou [ \pm animado] daquilo que estes produtos denotam decorre de condicicionalismos convencionais de natureza referencial/cultural, previstos na própria operação semântica da RFP AG (=agentiva) (1998: 119)².

De acuerdo con la argumentación de Rio-Torto, parece razonable hablar de un solo sufijo $-d o r$, asociado a la relación categorial $\mathrm{V} \rightarrow \mathrm{S}$ y a los significados agentivo e instrumental, que serían variantes condicionadas según que el nombre en -dor designe a una persona o un 
objeto $^{3}$. Esta propuesta viene corroborada por estudios como los de Laca (1986) y (1993), Levin-Rappaport (1992) o Gràcia (1995), entre muchos otros, donde se comprueba que, en realidad, el derivado en - dor incorpora el argumento que corresponde al sujeto del verbo base en una construcción activa, en otras palabras, el argumento externo de dicho verbo, que puede ser agente, instrumento, causa, experimentador, etc.

En (8) está representada la situación en que a una misma forma afijal corresponden varias relaciones categoriales y un solo significado. Como muestra hemos tomado el sufijo $-i z-a-r$, el equivalente del francés -is(er) con el que ejemplifica Corbin (1987: 249-252) la misma situación. Estos verbos indican "cambio de estado" y se pueden construir sobre una base adjetiva (ágil $\rightarrow$ agilizar) o nominal: (vapor $\rightarrow$ vaporizar). En el primero caso el verbo es parafraseable por "hacer (más) ágil"; en el segundo, por "transformar o convertir en vapor".

Si mantenemos el requisito de la unicidad en la relación categorial, -izar recubre dos sufijos homónimos asociados cada uno a una relación categorial diferente $(\mathrm{A} \rightarrow \mathrm{V}$ y S $\rightarrow$ V); pero, como observa Corbin, se pierde la relación semántica existente entre "hacer (más) A" y "transformar en S", paráfrasis ambas que están comprendidas en la noción genérica "cambio de estado". La solución alternativa es considerar que no hay más que un sufjo -izar asociado a una sola RFP; en este caso, o bien se intenta reducir las dos relaciones categoriales a una sola, o bien se admite el hecho de que la relación categorial sea múltiple, lo cual supone incumplir uno de los requisitos establecidos: la relación categorial única. La propuesta de Corbin (1987: 250), que consiste en reducir todos los verbos en -is(er) a una relación categorial única $A \rightarrow V$ con el significado "rendre (plus) Adj.", resulta a todas luces artificial y ad hoc. A falta de argumentos más convincentes que los aportados por Corbin, parece necesario admitir que en la base de estos verbos pueden estar nombres o adjetivos, y atribuir a la categoría nominal o adjetiva de la palabra base las variantes de significado que presentan los verbos derivados en -izar.

Está comprobado que el requisito de la relación categorial única -que no es otra cosa que la 'hipótesis de la base unitaria' (HBU) de Aronoff (1976) combinada con la 'hipótesis del educto único'- resulta demasiado fuerte porque, aparte de no cumplirse siempre en la sufijación heterogénea, resulta inadecuado en la sufijación homogénea (comp. - et-en amigo $\rightarrow$ amiguete, redondo $\rightarrow$ redondete, tocar $\rightarrow$ toquetear) y en la prefijación (comp. ante- en proyecto $\rightarrow$ anteproyecto, penúltimo $\rightarrow$ antepenúltimo, poner $\rightarrow$ anteponer), subsistemas donde la afijación es transversal a las distintas clases léxicas de palabras y donde, salvo en algunos casos de prefijación, la palabra derivada pertenece a la misma clase que la palabra base de derivación ${ }^{4}$. De ahí que, como es sabido, la HPU haya sido reformulada no sobre la base de categorías sintácticas, sino sobre la base de rasgos sintácticos de acuerdo con la teoría X barra. Según esta nueva hipótesis, denominada 'hipótesis de la base unitaria modi-

3 Argumentación y propuesta similar es la de Corbin (1987: 247-249) para los nombres sufijados en -oir(e) con los significados "instrumental" y "locativo". Rainer (1993: 218), por su parte, opta por mantener diferenciados los dos significados en el sufijo - dor.

4 Corbin (1987: 254), a propósito del análisis del prefijo dé-y del sufijo - ass (e), reconoce que el principio de la relación categorial única quizá no resulte válido para un subconjunto de los hechos derivativos. Según Rio-Torto (1998: 156), Corbin al final también acabó por cuestionar el principio de la relación categorial única: "Também nas versões mais recentes do pensamento da autora o princípio da unicidade categorial veio a ser posto em causa, tendo sido preterido em favor de um princípio de unicidade exclusivamente semântica". 
ficada' (HBUM) (Scalise, 1984: 139), un afijo se puede adjuntar a nombres y a adjetivos por compartir el rasgo $[+\mathrm{N}]$ o a adjetivos y a verbos por compartir el rasgo [+V], pero no a nombres y a verbos pues no comparten ninguno de los dos rasgos sintácticos categoriales ${ }^{5}$.

Hay que partir, pues, de una noción de paradigma derivativo menos restringida que la formulada inicialmente por Corbin en el sentido de que la $\mathrm{RC}$ no tiene por qué ser única. ¿Con qué nos encontramos desde esta perspectiva? Con la existencia de sufijos sinónimos y homónimos. En otras palabras y considerando al tiempo el afijo y la palabra derivada construida con dicho afijo, las situaciones que se nos presentan basculan entre los dos polos opuestos: la concurrencia de palabras derivadas o afijos con un mismo significado (sinonimia) y la concurrencia de significados diferentes en una palabra derivada o afijo (homonimia) ${ }^{6}$.

En efecto, como hemos visto en (6) a propósito de los sustantivos deadjetivales, dentro del mismo paradigma derivativo pueden figurar dos o más palabras derivadas sobre la misma base y con el mismo significado. A los ejemplos de (6) pueden añadirse otros similares donde se muestra la tendencia a crear neologismos con el mismo significado que los ya existentes; compárense los neologismos absurdez, concretidad o tontez frente a los nombres existentes absurdidad, concretez, tontedad $\sim$ tonteria.

Por otro lado, ocurre que dos o más tipos de significado correspondientes a distintos paradigmas o RFP coexisten en una palabra derivada o en un afijo sencillamente porque la misma forma afijal figura en más de un paradigma derivativo. Por poner un ejemplo, dentro del subsistema de los nombres denominales hay varios paradigmas, entre ellos, el paradigma de los nombres "colectivos" mostrado en (4) y el de los nombres que indican "acto propio de" en:

(9)

$\begin{array}{lll}\text { afijo } & \mathrm{RC} & \text { significado } \\ \text {-ada } & \mathrm{S} \rightarrow \mathrm{S} & \text { acto propio de } \\ \text { - eria } & \mathrm{S} \rightarrow \mathrm{S} & \text { acto propio de }\end{array}$

bribonada, cacicada niñeria, haraganeria

Si se contrastan ambos paradigmas se verá que los dos sufijos del paradigma de los nombres que significan "acto propio de" están también presentes en el paradigma de los nombres colectivos. De ahí que figuren en la lengua nombres en -ada y, sobre todo, nombres en -eria (por ser el sufijo más rentable en la formación de nombres colectivos) que expresan ambas nociones: granuja $\rightarrow$ granujeria 'conjunto de granujas' y 'acto propio de un granuja', chiquilleria "conjunto de chiquillos" y "acto propio de chiquillos", y lo mismo chuleria, gallegada, gitaneria, golferia, judiada, mamarrachada, muchachada muchacheria, paletería, truhanada $\sim$ truhanería, etc.

5 Como es sabido, en la teoría de la $\mathrm{X}$ barra las categorías verbo, nombre, adjetivo y preposición pueden analizarse en términos de los rasgos categoriales sintácticos $\mathrm{N}$ (nombre) y $\mathrm{V}$ (verbo) como sigue: verbo [ $+\mathrm{V},-\mathrm{N}]$, adjetivo $[+\mathrm{V},+\mathrm{N}]$, nombre $[-\mathrm{V},+\mathrm{N}]$, preposición $[-\mathrm{V},-\mathrm{N}]$. Tal sistema de rasgos permite las generalizaciones siguientes: $\mathrm{V}$ y $\mathrm{P}$ se caracterizan como [-N], $\mathrm{N}$ y $\mathrm{P}$ como [-V], A y $\mathrm{N}$ como [+N ], V y A como [+V ].

6 El criterio utilizado por Corbin para distinguir la homonimia de la polisemia en el dominio de la palabra deirvada es el siguiente:

si à une structure morphologique formellement unique correspondent plusieurs sens superficiellement différents et nécessairement compositionnels, il y a polysémie si les sens sont dérivables sémantiquement l'un de l'autre, homonymie s'ils ne le sont pas. Si l'un des sens n'est pas compositionnel, il y a homonymie. 
De acuerdo con lo expuesto hasta aquí, el requisito necesario para adscribir uno o más afijos a un mismo paradigma es el de la unicidad semántica, esto es, que expresen un solo significado; si una misma forma afijal expresa más de un significado, estamos ante dos o más afijos homónimos. Ahora bien, ¿es necesario mantener estrictamente este requisito? Probablemente no. El problema está, como ya queda indicado, en saber cuándo dos (o más) significados presentes en un afijo son reducibles a uno solo o no. Hay una situación, al menos, en la que la presencia de más un significado en el afijo no hay por qué interpretarla necesariamente como homonimia afijal. Ocurre cuando la coexistencia de dos o más significados puede describirse mejor pensando en un significado nuclear o básico del que derivan otros significados secundarios, que recurriendo a un significado abstracto invariante que comprende o abarca los diferentes significados particulares. Esta es la propuesta que hace, por ejemplo, Zwanenburg (1984a: 132) cuando afirma:

The different meanings which a complex word may have must be described as a core meaning plus a set of derived meanings rather than as a general abstract meaning covering the different particular meanings it may have ${ }^{7}$

Efectivamente, esta situación donde, a partir del significado básico, la palabra puede adquirir significados secundarios derivados de él por medio de desplazamientos metonímicos fundamentalmente es frecuente en las lenguas y es un fenómeno semántico y no estrictamente morfológico porque se da igualmente en las palabras simples y derivadas. $\mathrm{Si}$ retomamos el paradigma de los nombres de cualidad presentado en (5), se puede comprobar que el sustantivo de cualidad puede expresar, en coexistencia con el significado regular "cualidad de", otros significados derivados de él ${ }^{8}$. Así, en una determinada dirección, del significado "cualidad de A" se puede pasar al significado "actitud de (quien es) A": amabilidad "cualidad de amable" (esa amabilidad que nace de nuestro deseo de complacer) y "actitud (de quien es) amable" (la amabilidad que mostró al recibirte). En una segunda dirección, mediante el cambio de sustantivo abstracto a sustantivo concreto, se pasa de la "cualidad de A" a la entidad portadora de esa cualidad o a la circunstancia en que ocurre esa cualidad: novedad "cualidad de nuevo" (la novedad de este método) y "cosa nueva" (las últimas novedades en artículos estampados); ancianidad "cualidad, condición de anciano" (su ancianidad no le impide trabajar) y "período de vida que abarca la ancianidad" (pasar la ancianidad entre los seres queridos es lo más bonito $)^{9}$, etc.

\section{Final}

A lo largo del presente artículo he ido presentando los distintos niveles donde cabe observar la relación derivativa y viendo las dificultades que surgen así como algunas de las

\footnotetext{
7 Esta tesis, que aparece desarrollada en Zwanenburg (1984a: 132-134), es reproducción literal de la que aparece en Zwanenburg (1980: 334-336).

8 Este fenómeno ha sido bastante estudiado, aunque con resultados poco satisfactorios hasta el momento. Los significados secundarios derivados reciben distintas denominaciones: extensiones semánticas, polisemia regular, transferencias de sentido, desplazamientos semánticos, etc. Vid. Nunberg-Zaenen (1997). Para las extensiones semánticas sigue siendo de capital importancia el capitulo VIII de Kany (1960).

9 Ejemplos tomados de los diccionarios Clave, dirigido por C. Maldonado, y Salamanca, dirigido por J. Gutiérrez.
} 
soluciones adoptadas. Al final (3.1) he desarrollado y matizado lo que se conoce como "el modelo asociativo débil' basándome fundamentalmente en la propuesta de Corbin (1987).

Que me haya detenido en la exposición de una variante moderada del modelo asociativo no quiere decir, ni mucho menos, que descarte la validez del modelo disociativo. Es más, en mi opinión, el presentar ambos modelos como antitéticos responde a un falso problema, porque no son excluyentes sino complementarios. En la morfología derivativa se pueden y se deben estudiar por separado los procedimientos formales y la estructura de los significados codificados mediante tales procedimientos, teniendo siempre presente que son los significados en sí los que están organizados estructuralmente y no los procedimientos, que son las manifestaciones garantes de la distinción entre significados. Pero nada impide que en una segunda fase se estudien conjuntamente procedimientos y significados, bien desde el punto de vista de la expresión, bien desde el punto de vista del contenido.

La posibilidad de estudiar conjuntamente las relaciones formales y semánticas viene dada por la propia naturaleza de la morfología derivativa: la existencia de palabras relacionadas formal y semánticamente. Dicho con más precisión, hay dos propiedades que caracterizan a la morfología derivativa y que la acercan a la gramática al tiempo que la alejan de la lexicología. En primer lugar, las diferencias entre los significados codificados mediante derivación son recurrentes: al igual que la diferencia "singular / plural" existente en casa / casas se repite en libro / libros, metro / metros, etc., también la diferencia entre "verbo" y "argumento externo del verbo" que aparece en animar / animador, ganar / ganador, nadar I nadador, etc. En segundo lugar, las oposiciones entre significados presentan una proporcionalidad con las diferencias de sus significantes (Coseriu, 1981: 57); el grado máximo de regularidad se presenta en la flexión, pero también aparece en la derivación: si en casa / casas y mesa / mesas, hay recurrencia en el significante y en el significado, también la hay en animar / animador y nadar / nadador, y los hechos de alomorfía aparecen tanto en la flexión como en la derivación: casa / casas, cordel / cordeles; animar / animador, conducir / conductor. Ambas propiedades son las que permiten estudiar conjuntamente la forma y el significado de las palabras derivadas, a pesar de existencia de numerosos casos de correspondencia multívoca. En este sentido, el modelo asociativo débil formulado por Corbin parece bastante adecuado a la realidad de los hechos, siempre que se elimine de la noción de paradigma derivativo el requisito de la relación categorial única entre la palabra base y la derivada. En este modelo es el significado el que aglutina en un mismo paradigma los diferentes procedimientos, del mismo modo que en gramática es el significado "plural" el que une sus diferentes formas de expresión: - $-s$ (casas) o -es (cárceles).

\section{Referencias bibliográficas}

Beard, R. (1984a): "Generative Lexicalism", Quaderni di Semantica, V/1, págs. 50-57.

Beard, R. (1984b): "Optimism, Pessimism and Finer Distinctions", Quaderni di Semantica, V/2, págs. 277-287.

Becker, Th. (1993): "Back-formation, cross-formation, and 'bracketing paradoxes' in paradigmatic morphology". En Geert Boij y Jaap van Marle (eds.): Yearbook of Morphology 1993. Netherlands, Kluwer Academic Publishers, págs. 1-25.

Bosque, I. y V. Demonte (dirs.) (1999): Gramática descriptiva de la lengua española. Real Academia Española, Colección Nebrija y Bello, Madrid, Espasa Calpe. 
Corbin, D. (1987): Morphologie dérivationnelle et structuration du lexique, 2 vols. Tubinga, Max Niemeyer Verlag, segunda parte, cap. 2, apdo. 5: "Distorsions apparentes entre la forme et le sens", págs. 208-282.

Coseriu, E. (1981): "Para una semántica diacrónica estructural". En Principios de semántica estructural. Madrid, Gredos, págs. 11-86.

Flaux, N. et alii (eds.) (1996): Les noms abstraits. Histoire et théories. Paris, Presses Universitaires du Septentrion.

Gràcia i Solé, Ll. (1995): Morfologia lèxica. L'herència de l'estructura argumental. València, Universitat de València.

Gutiérrez Cuadrado, J. (dir.) (1996): Diccionario Salamanca de la lengua española. Madrid, Santillana-Universidad de Salamanca.

Jackendoff, R. (1975): "Morphological and semantic regularities in the lexicon", Language, 51, págs. 639-671. Traducción castellana (1979): "Regularidades morfológicas y semánticas en el lexicón". En Chomsky et alii: La teoría estándard extendida. Madrid, Cátedra, págs. 72-116.

Kany, Ch. (1960): Semántica hispanoamericana. Madrid, Aguilar, 1969.

Laca, B. (1986): Die Wortbildung als Grammatik des Wortschatzes. Untersuchungen zur spanischen Subjektnominalisierung. Tubinga, Narr.

Laca, B. (1993): "Las nominalizaciones orientadas y los derivados españoles en - dor y $-n t e "$. En Soledad Varela (ed.): La formación de palabras. Madrid, Taurus Universitaria, págs. 180-204.

Levin, B. y M. Rappaport (1992): "-Er nominals: implications for the theory of argument structure". En Tim Stowell y Eric Wehrli (eds.): Syntax and Semantics, vol. 26: Syntax and Lexicon. California, Academic Press, págs. 127-152.

Lieber, R. (1980): On the Organization of the Lexicon (Tesis doctoral, MIT). Bloomington, Indiana Univ. Linguistic Club.

Maldonado González, C. (dir.) (1996): Clave. Diccionario de uso del español actual. Madrid, Ediciones SM.

Nunberg, G. y A. Zaenen (1997): "La polysémie systématique dans la description lexicale”, Langue Française, 113, págs. 12-23.

Pena, J. (1990): "Sobre los modelos de descripción en morfología", Verba, 17, págs. 5-75.

Rainer, F. (1993): Spanische Wortbildungslehre. Tubinga, Max Niemeyer Verlag.

Rainer, F. (1999): "La derivación adjetival". En Bosque I. y V. Demonte (dirs.): Gramática descriptiva de la lengua española. Real Academia Española, Colección Nebrija y Bello, Madrid, Espasa Calpe, vol. 3, págs. 4595-4643.

Rio-Torto, G. M. (1998): Morfologia derivacional. Teoria e Aplicação ao Português. Portugal, Porto Editora.

Scalise, S. (1984): Generative morphology. Dordrecht, Foris.

Spencer, A. (1991): Morphological Theory. Oxford, Blackwell.

Zwanenburg, W. (1980): "Form and Meaning in Morphology", Quaderni di semantica, 2, págs. 327336.

Zwanenburg, W. (1984a): "Word formation and meaning", Quaderni di Semantica, V/1, págs. 130142.

Zwanenburg, W. (1984b): "Word Formation and meaning: Derivation Types", Quaderni di Semantica, V/2, págs. $350-365$. 